\title{
茶汤抗氧化活性的测定综合化学实验的研究
}

\author{
严赞开 ${ }^{*}$, 郑丽玲 \\ 韩山师范学院化学与环境工程学院, 广东潮州 521041
}

\begin{abstract}
摘要: 实验以潮州工夫茶文化为背景, 在工夫茶传统冲泡工艺的基础上, 采用茶汤抗氧化活性为评判指标, 探讨工夫茶 冲泡工艺的优化条件。实验内容包括文献调研、实验方案设计、实验操作、数据处理及小论文撰写等一系列学习任务。 实践教学结果表明, 将传统茶文化融入实验教学中, 易于激发学生的学习兴趣, 传递文化基因; 引导学生从文化生活源 头提出问题, 运用科学方法分析问题、解决问题, 利于学生熟悉科学过程, 养成正确的科学思维习惯; 同时, 实践活动 中学生会触及到多个学科领域、多重实验操作技能训练, 可以全面提升学生的综合素养, 达成课程育人目标。
\end{abstract}

关键词: 综合性; 化学实验; 抗氧化活性

中图分类号: G64; O6

\section{Study on a Comprehensive Chemical Experiment: Determination of Antioxidant Activity of Tea Soup}

\author{
Zankai Yan *, Liling Zheng \\ Department of Chemistry and Environmental Engineering, Hanshan Normal University, Chaozhou 521041, Guangdong Province, \\ China.
}

\begin{abstract}
Taking Chaozhou Gongfu tea culture as the background, based on the traditional brewing technology of Gongfu tea, the antioxidant activity of tea soup was used as the evaluation index to explore the optimization conditions of brewing technology of Gongfu tea. The content of the experiment includes a series of learning tasks, such as literature research, experimental scheme design, experimental operation, data processing and essay writing. The results of practical teaching show that the integration of traditional tea culture into experimental teaching is easy to stimulate students' interest in learning and transfer cultural genes. To guide students to put forward problems from the source of cultural life, to analyze and solve problems with scientific methods will help students to be familiar with the scientific process and develop correct habits of scientific thinking. At the same time, students in practical activities will touch on multiple disciplines and multiple laboratory skills training, which can comprehensively improve students' comprehensive quality and achieve the goal of curriculum education.
\end{abstract}

Key Words: Comprehensive; Chemical experiment; Antioxidant activity

茶在中国有着非常悠久的历史, 就茶的冲泡而言, 潮州的工夫茶堪称一绝 ${ }^{[1,2]}$ 。在冲泡过程中, 茶叶中的主要抗氧化活性成分茶多酚不断溶出, 长期饮茶则有益于身体健康 ${ }^{[3]}$ 。就工夫茶的茶汤品 质而言, 传统的评价方式是感观指标, 由于饮茶人对茶汤的浓烈、淡雅存在不同的喜好, 自然评价 标准不一, 导致冲泡茶时工艺条件因人而异。论茶的沏泡技艺, 古代有斗茶文化, 今天我们又如何

收稿: 2021-04-16; 录用: 2021-06-17; 网络发表: 2021-07-06

“通讯作者, Email: yanxiao4300@163.com

基金资助: 广东省教育厅高等教育教学改革项目(jg092) 
科学饮茶呢? 带着这个问题, 本文以潮州工夫茶文化为背景, 介绍一个以茶汤抗氧化活性为评判指 标, 优化冲泡条件的综合化学实验。首先指导学生收集民间泡茶的工艺条件, 制订出优化茶冲泡的 工艺方案; 其次通过查阅科学文献资料, 设计出茶汤抗氧化活性的测定步骤, 并开展实验; 最后分 析实验数据, 得出茶汤的抗氧化活性与冲泡工艺条件间的相关规律, 撰写小论文。整个实验项目集 化学、食品、生物等学科知识为一体, 学生经历文献检索、方案设计、实验操作、数据分析、撰写 论文等诸多环节的一体化训练, 能有效地发展学生的综合实践素养, 达成课程育人目标。

\section{1 实验目的}

（1）通过文献调研，了解工夫茶文化，收集民间冲泡茶的工艺条件;

(2) 熟悉植物多酚抗氧化活性的测定方法及原理, 并设计出实验方案;

(3) 掌握分光光度计的操作技术及样品的采集、分析, 数据处理等方法;

(4) 养成敢于面对困难, 严谨务实、精益求精的科学态度。

\section{2 实验原理}

(1) 还原能力的测定: 以普鲁士蓝 $\mathrm{Fe}_{4}\left[\mathrm{Fe}(\mathrm{CN})_{6}\right]$ 生成量作为指标。抗氧化剂将赤血盐 $\mathrm{K}_{3} \mathrm{Fe}(\mathrm{CN})_{6}$ 还 原成黄血盐, 再利用 $\mathrm{Fe}^{2+}$ 形成普鲁士蓝, 在 $700 \mathrm{~nm}$ 处的吸光值反映普鲁士蓝的生成量, 吸光度值越 高, 样品还原力(抗氧化能力)愈强, 表示抗氧化效果愈佳。

(2) 清除DPPH (1,1-二苯基-2-苦肼基)的测定: DPPH·乙醇溶液呈紫色, 在 $517 \mathrm{~nm}$ 有强吸收, 加 入抗氧化剂后, 抗氧化剂与 DPPH. 反应, 使反应体系颜色变淡, 吸光度 $A$ 值减少, 由此可计算抗氧化 剂清除DPPH·的能力。

(3) 清除· $\mathrm{OH}$ 能力的测定: 反应生成的· $\mathrm{OH}$ 进攻水杨酸分子上的苯环, 生成有色产物, 在 $510 \mathrm{~nm}$ 波长处有较强吸收, 反应体系中若加入具有清除. $\mathrm{OH}$ 作用的物质可降低该吸光度。因此, 通过测定有 色物质含量来测定抗氧化剂清除· $\mathrm{OH}$ 的能力。

(4) 清除 $\cdot \mathrm{O}_{2}^{-}$能力的测定: 在弱碱性环境下, 邻苯三酚发生自氧化反应, 不断生成 $\cdot \mathrm{O}_{2}^{-}$(超氧阴离 子自由基)及有色中间产物。抗氧化剂将 $\mathrm{O}_{2}^{-}$歧化分解为 $\mathrm{H}_{2} \mathrm{O}_{2}$ 和 $\mathrm{O}_{2}$, 从而抑制了邻苯三酚的自氧化反 应。中间产物累积浓度会引起波长 $325 \mathrm{~nm}$ 处吸光度值的变化, 由此可测得清除 $\cdot \mathrm{O}_{2}^{-}$的能力。

\section{3 仪器与材料}

(1) 仪器: $752 \mathrm{~N}$ 型紫外可见分光光度计(上海棱光技术有限公司), FA2004N型电子天平(上海精 密科学仪器有限公司), 数显恒温水浴锅(常州市华谱达教学仪器有限公司), SJT-018A电热壸(潮州市 彩塘适特电器配件厂)。

(2) 试剂: 1,1-二苯基-2-苦肼基DPPH (1,1-diphenyl-2-picryl-hydrazyl), 无水乙醇, 硫酸亚铁, $\mathrm{H}_{2} \mathrm{O}_{2}$, 水杨酸, 铁氭化钾, 三氯乙酸, 三氯化铁, 磷酸氢二钠, 磷酸二氢钾, 盐酸, 碳酸钠等, 以上试剂 均为分析纯试剂, 由潮州市化学试剂公司供货。

（3）材料：黄枝香风凰单枞, 当年春茶, 产自广东省潮州市风凰山区。

\section{4 实验步骤}

\section{1 茶汤样品的制备}

(1) 料液比: 准确称取 $0.50 \mathrm{~g}$ 茶叶 6 份, 分别按固液比 $1: 50 、 1: 100 、 1: 150 、 1: 200 、 1: 250$ 、 $1: 300\left(\mathrm{~g} \cdot \mathrm{mL}^{-1}\right)$ 加入 $90{ }^{\circ} \mathrm{C}$ 的蒸馏水, 在 $90^{\circ} \mathrm{C}$ 下保温 $30 \mathrm{~min}$, 过滤, 收集滤液, 即得待测液。

(2) 冲泡温度: 准确称取 $0.50 \mathrm{~g}$ 茶叶6份, 按固液比 $1: 200\left(\mathrm{~g} \cdot \mathrm{mL}^{-1}\right)$ 分别加入 $60 、 70 、 80 、 90$ 、 $100{ }^{\circ} \mathrm{C}$ 的蒸馏水, 按加入的水温各保温 $30 \mathrm{~min}$, 过滤, 收集滤液, 即得待测液。

(3) 冲泡时间: 准确称取 $0.50 \mathrm{~g}$ 茶叶 6 份, 按固液比 $1: 200\left(\mathrm{~g} \cdot \mathrm{mL}^{-1}\right)$ 都加入 $90{ }^{\circ} \mathrm{C}$ 的蒸馏水 $100 \mathrm{~mL}$, 
在 $90{ }^{\circ} \mathrm{C}$ 下分别保温10、20、30、40、50、60 min，过滤，收集滤液，即得待测液。

\section{2 茶汤抗氧化活性的测定}

(1) 茶汤还原力测定: 按参考文献 ${ }^{[4]}$ 的方法, 将采用稀释 10 倍的茶汤作为样品。精确移取 $2.50 \mathrm{~mL}$ 样品液, 向其中加入磷酸缓冲溶液 $\left(0.2 \mathrm{~mol} \cdot \mathrm{L}^{-1}, \mathrm{pH}=6.6\right) 、 1 \%$ 铁氰化钾溶液各 $2.50 \mathrm{~mL}$, 在 $50{ }^{\circ} \mathrm{C}$ 下 保温水浴 $20 \mathrm{~min}$ 后, 冰浴终止反应。加 $10 \%$ 三氯乙酸溶液 $2.50 \mathrm{~mL}, 3000 \mathrm{r} \cdot \mathrm{min}^{-1}$ 离心 $10 \mathrm{~min}$, 取上清 液 $2.50 \mathrm{~mL}$, 再加 $2 \mathrm{~mL}$ 蒸馏水和 $0.1 \%$ 三氯化铁溶液 $0.50 \mathrm{~mL}$, 静置 $10 \mathrm{~min}$, 在 $700 \mathrm{~nm}$ 波长处测定吸光 度 $(A)$, 每组试验平行 3 次, 实验结果取平均值。计算总还原力的公式如下:

$$
A_{\text {总还原力 }}=A_{\text {样品 }}-A_{\text {空白 }}
$$

(2) 清除DPPH·能力测定: 按参考文献 ${ }^{[5]}$ 中的方法, 采用稀释 10 倍的茶汤作为样品。准确称取 $0.1984 \mathrm{~g} \mathrm{DPPH}$, 用无水乙醇溶解后定容至 $50 \mathrm{~mL}$ 用作储备液。吸取DPPH储备液 $2 \mathrm{~mL}$, 用无水乙醇定 容至 $100 \mathrm{~mL}$ 。分别吸取 $2 \mathrm{~mL} \mathrm{DPPH}$ 溶液和 $2 \mathrm{~mL}$ 无水乙醇于试管中, 用力摇匀, 避光反应 $30 \mathrm{~min}$, 在 波长 $517 \mathrm{~nm}$ 处测定其吸光值 $\left(A_{0}\right)$ 。另取试管, 用 $2 \mathrm{~mL}$ 茶汤替代无水乙醇, 测定其吸光值 $\left(A_{i}\right)$; 再取试 管, 用 $2 \mathrm{~mL}$ 茶汤替代DPPH溶液, 测定其吸光值 $\left(A_{j}\right)$ 。每组试验平行 3 次, 实验结果取平均值。计算 DPPH的清除率公式如下:

$$
\text { PPH清除率 }=\frac{A_{0}-\left(A_{i}-A_{j}\right)}{A_{0}} \times 100 \%
$$

(3) 茶汤清除. $\mathrm{OH}$ 能力测定: 按参考文献 ${ }^{[6]}$ 的方法进行实验, 将采用稀释 10 倍的茶汤作为样品。 精确吸取 $1 \mathrm{~mL} \mathrm{FeSO} 4\left(9 \mathrm{mmol} \cdot \mathrm{L}^{-1}\right) 、 1 \mathrm{~mL}$ 水杨酸-乙醇 $\left(9 \mathrm{mmol} \cdot \mathrm{L}^{-1}\right)$ 和茶汤 $1 \mathrm{~mL}$ 。再加入 $1 \mathrm{~mL} \mathrm{H}_{2} \mathrm{O}_{2}$ $\left(8.8 \mathrm{mmol} \cdot \mathrm{L}^{-1}\right)$, 在 $37^{\circ} \mathrm{C}$ 下保温反应 $0.5 \mathrm{~h}$, 在波长 $510 \mathrm{~nm}$ 处测定吸光度 $\left(A_{\mathrm{x}}\right)$ 。计算 $\cdot \mathrm{OH}$ 清除率的公式 如下:

$$
\text { -OH清除率 }=\frac{A_{0}-\left(A_{\mathrm{x}}-A_{\mathrm{x} 0}\right)}{A_{0}} \times 100 \%
$$

式中: $A_{0}$, 样品空白时吸光度; $A_{\mathrm{x}}$, 加入茶样后吸光度; $A_{\mathrm{x} 0}$, 不加显色剂 $\mathrm{H}_{2} \mathrm{O}_{2}$ 、加茶样的吸光 度。

(4) 茶汤清除超氧阴离子测定: 参考文献 ${ }^{[7]}$ 的方法, 将采用稀释 10 倍的茶汤作为样品。取 $2.50 \mathrm{~mL}$ 、 $50 \mathrm{mmol} \cdot \mathrm{L}^{-1}$ 的磷酸盐缓冲溶液 $(\mathrm{PBS}, \mathrm{pH}=8.34$ ), 加入 $2 \mathrm{~mL}$ 样品溶液, 再加入 $0.25 \mathrm{~mL}$ 的邻苯三酚 $\left(10 \mathrm{mmol} \cdot \mathrm{L}^{-1}\right)$, 在 $25{ }^{\circ} \mathrm{C}$ 下保温 $4 \mathrm{~min}$, 加入 $10 \mathrm{mo} \cdot \mathrm{L}^{-1} \mathrm{HCl} 1$ 滴, 终止反应; 用相同的样品液为参比, 在波长 $325 \mathrm{~nm}$ 处测其吸光度值, 得到样品的吸光度值 $\left(A_{1}\right)$ 。用蒸馏水代替样品溶液, 加入与测试样品 相同的其它试剂, 以 $\mathrm{pH}=8.34$ 的PBS溶液做参比, 在波长 $325 \mathrm{~nm}$ 处测其吸光度值, 得到空白吸光度 值 $\left(A_{0}\right)$ 。计算超氧阴离子的消除率的公式如下:

$$
\text { 超氧阴离子清除率 }(\%)=\frac{A_{0}-A_{1}}{A_{0}} \times 100 \%
$$

\section{5 结果与讨论}

\section{1 茶水比对茶汤抗氧化活性的影响}

在茶水比对茶汤的抗氧化能力的考查实验中, 设定冲泡温度 $90{ }^{\circ} \mathrm{C} 、$ 冲泡时间 $30 \mathrm{~min}$ 的条件不变, 过滤后得到原始茶汤。由于固液比不同, 原始茶汤浓淡不同, 直接取样实验, 茶汤的抗氧化能力体 现的只是原始茶汤单位体积的抗氧化能力, 即茶汤的局部抗氧化能力。这样的比较是片面的, 只反 映了茶汤局部浓淡的作用, 只有将茶汤定容, 所有茶汤样的体积一致, 这时取样比较, 才可以反映 茶汤的总体抗氧化能力。

为了弄清固液比对茶汤总的抗氧化能力的影响, 实验将全部的原始茶汤先定容至 $300 \mathrm{~mL}$, 再稀 释10倍, 在等同体积下进行比较, 即茶汤总抗氧化能力的比较, 实验结果见图 1 和图2。从实验结果 
可知, 随固液比的比值增大, 茶汤的总体还原能力不断上升。而对自由基的清除实验则出现各自的 特点, DPPH·的清除率随固液比一直上升, 但在 $1: 200\left(\mathrm{~g} \cdot \mathrm{mL}^{-1}\right)$ 后明显变缓; $\mathrm{O}_{2}^{-}$的清除率随固液比的 比值增大, 在 $1: 250\left(\mathrm{~g} \cdot \mathrm{mL}^{-1}\right)$ 处出现一个峰值; $\cdot \mathrm{OH}$ 则在 $1: 150\left(\mathrm{~g} \cdot \mathrm{mL}^{-1}\right)$ 处出现峰值。综合几个因素 的考核结果, 可以得出: 固液比在 $1: 150-1: 250\left(\mathrm{~g} \cdot \mathrm{mL}^{-1}\right)$ 范围内, 所得茶汤总体抗氧化能力的较强。

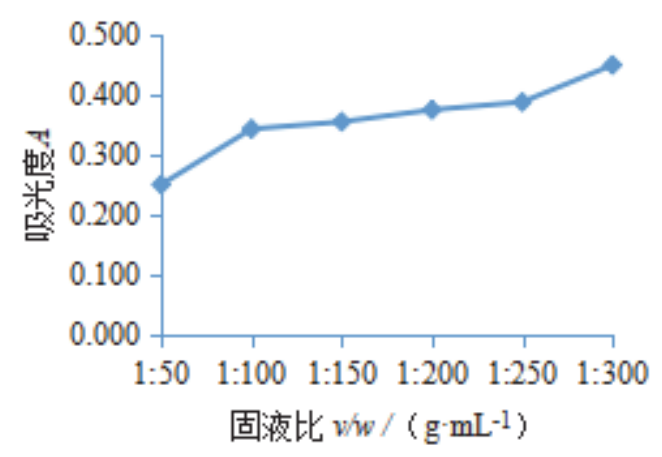

图1 固液比对茶汤总还原力的影响

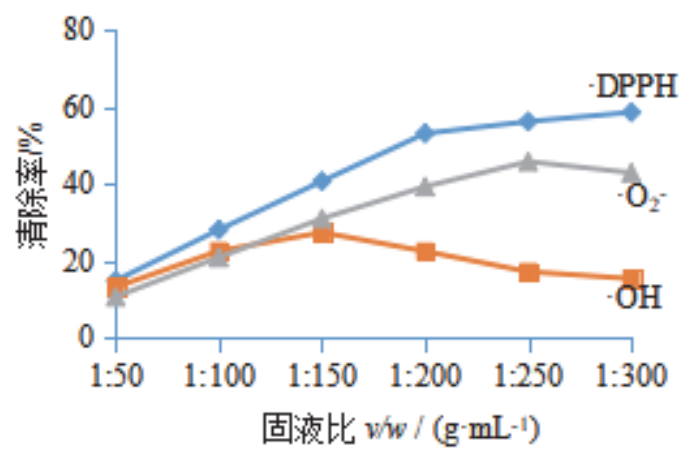

图2 固液比对茶汤自由基清除率的影响

\section{2 冲泡时间对茶汤抗氧化活性的影响}

冲泡茶时的固液比选择 $1: 200\left(\mathrm{~g} \cdot \mathrm{mL}^{-1}\right)$, 冲泡温度固定为 $90{ }^{\circ} \mathrm{C}$, 实验结果见图3和图 4 。随着冲 泡时间的增加, 茶汤的还原力逐渐上升; 当时间超过 $40 \mathrm{~min}$ 后, 上升趋势变缓。羟基自由基的清 除曲线与还原能力曲线走势一致, 但清除自由基的能力较弱。在对 DPPH·和超氧阴离子自由基的清 除实验中, 当时间为 $40 \mathrm{~min}$ 时出现峰值, 形成一个倒 $\mathrm{V}$ 型走势。从实验结果可以看出, 当固液比 1 : $200\left(\mathrm{~g} \cdot \mathrm{mL}^{-1}\right)$, 冲泡温度 $90{ }^{\circ} \mathrm{C}$, 冲泡茶的时间在30-40 min时, 所得茶汤的抗氧化能力较强。

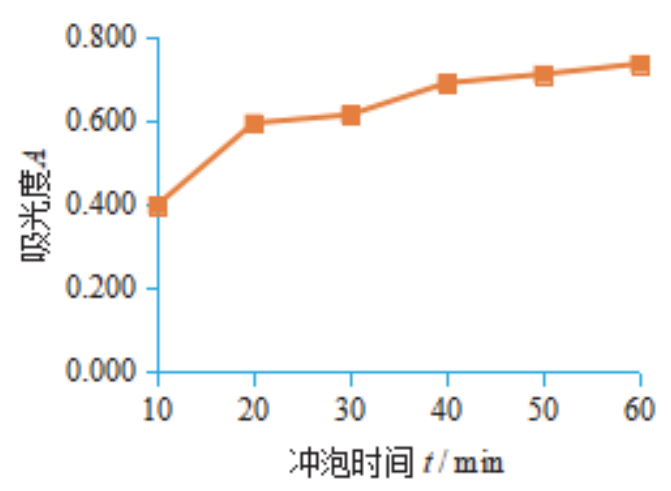

图3 冲泡时间对还原力的影响

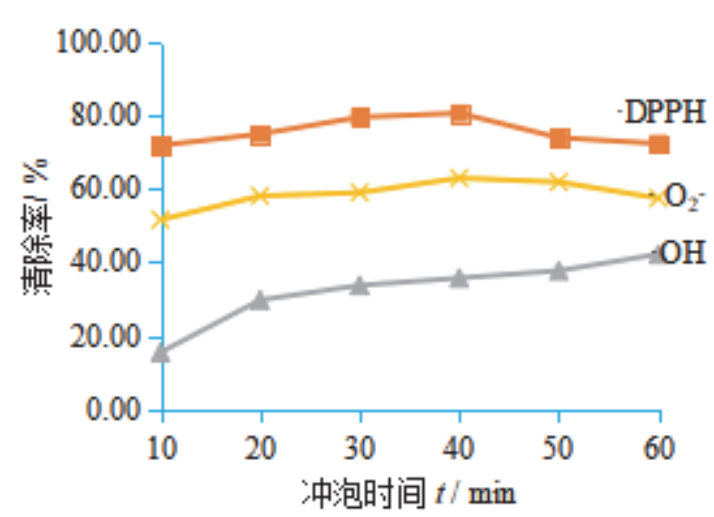

图4 冲泡时间对自由基清除率的影响

\section{3 冲泡水温对茶汤抗氧化活性的影响}

实验设定固液比 $1: 200\left(\mathrm{~g} \cdot \mathrm{mL}^{-1}\right)$, 冲泡时间为 $30 \mathrm{~min}$ 不变, 考查温度对茶汤抗氧化能力的影响, 实验数据见图 5 和图 6 。随着冲泡温度的升高, 茶汤的还原能力逐渐上升, 到 $90^{\circ} \mathrm{C}$ 时达到峰值。同样, 随着冲泡温度的升高, 自由基的清除率不断上升; 其中, $\mathrm{DPPH} \cdot$ 和 $\mathrm{O}_{2}^{-}$的清除率在 $90^{\circ} \mathrm{C}$ 时达到峰值, 而羟基自由基的清除率在 $90{ }^{\circ} \mathrm{C}$ 时接近峰值。从实验结构可知, 茶叶冲泡温度在 $90{ }^{\circ} \mathrm{C}$ 时茶汤的抗氧 化活性较强。

综合上述研究结果, 本实验通过冲泡茶提取茶汤, 以茶汤的抗氧化能力为指标, 得出工夫茶的 较优冲泡方法为: 按茶水固液比 $1: 200\left(\mathrm{~g} \cdot \mathrm{mL}^{-1}\right)$ 投料, 在温度 $90{ }^{\circ} \mathrm{C}$, 冲泡时间为 $30-40 \mathrm{~min}$ 时, 实验达 到预期研究目标。 


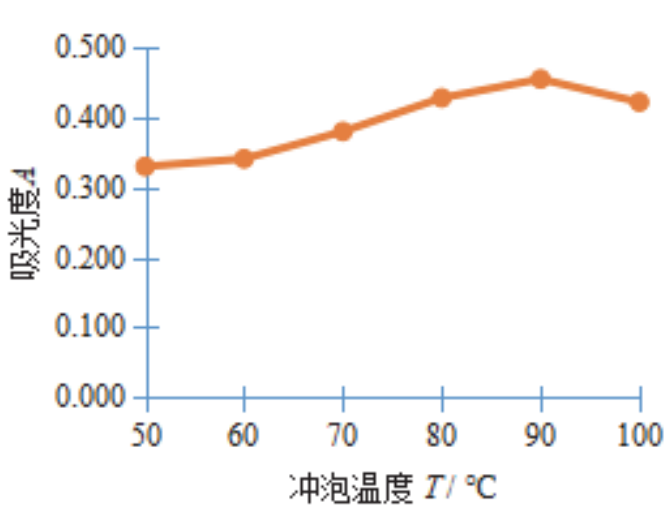

图5 冲泡温度对还原力的影响

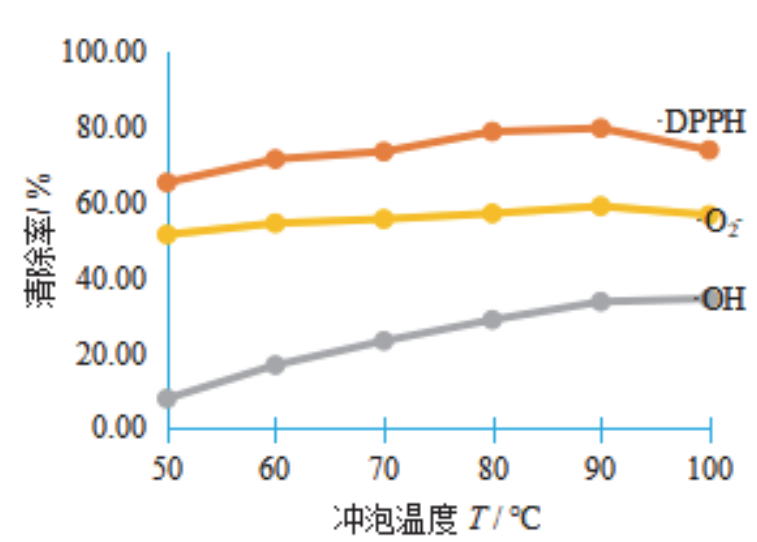

图6 冲泡温度对自由基清除率的影响

\section{6 实验运行情况}

为保证高质量完成实验教学任务, 应统筹安排好以下几个方面。

(1) 教学管理。本综合设计性实验是由教师的科研成果开发而成, 经学院教学委员会审核通过 后, 方对学生开课。教学采用科研项目管理的形式进行, 以学生为主体、教师为辅导。教师采用一 对一方式服务学生, 全程跟踪每位学生的实验过程, 确保达到预期的教学目标。

(2) 实验筹备。首先教师给学生讲明实验目的、教学内容、教学计划、质量要求等事项, 要求每 位学生制订一个自己的实验工作计划。其次要求学生查阅工夫茶相关的文献资料, 了解茶、茶文化、 冲泡茶相关的知识, 收集民间冲泡工艺的相关数据。其次在教师指导下, 学生查阅植物多酚抗氧化 活性的测定方法及原理的相关资料, 依据实验室条件, 撰写实验方案, 包括实验操作步骤、所需的 仪器及材料等。方案经教师审核通过后, 学生方可进入实验室开展实验前的准备工作。

(3) 预备实验。综合实验按一人一组、独立研究的形式进行。由于学生在上综合实验课之前, 只 接受过基础实验的训练。在基础实验中, 实验的准备工作及质量把关均由实验教师把控, 学生做实 验多以验证性体验为主。进入综合实验环节后, 小型实验仪器的调试、试剂的配制、实验的操作要 领等均由学生来把控。为保证实验教学质量, 在教师讲解了仪器的操作要领、试剂的配制、实验操 作规范及注意事项后, 学生要经过大量的预试实验, 反复比对实验数据。对于造成数据偏差大, 或 稳定性差的操作环节, 组织讨论, 分析疑点, 找出原因, 提出解决的方案, 再反复修正操作、比对 数据, 直到每个操作细节准确无误, 数据可靠、可信为止。

(4) 数据的采集与处理。在实验过程中, 依照预试的操作, 尽量减少仪器、操作、人为随机等因 素带来的偏差, 样品的处理、测定方法保持一致。如用恒温水浴锅替代茶壶, 可保证冲泡时温度稳 定; 又如冲泡所得的茶汤样品, 为了避免放置时间长氧化, 影响抗氧化活性测定结果, 都是即时处 理, 并控制每个样显色、测定时间一致。每个样平行测定三次, 取平均值计算。运用Excel软件对实 验数据计算、绘图, 找出实验结果的规律性。

(5) 论文的撰写。撰写论文对于大三学生来说, 有一定的难度。教师首先要拿一篇范文给学生讲 解论文由哪几部分组成, 每一部分有什么功能, 如何写好每一部分, 学生先模仿写作。经多次修改 后, 学生总算明白如何撰写文献综述, 来说明自己做实验的意义; 通过分析实验结果, 来说明实验 的创新点。其次要求学生严格修订文章的格式, 重点包括文字、图、表的格式规范, 参考文献标注 及参考文献的书写格式, 以及专业术语的表达等。

(6) 学生成绩评定。由于综合实验涉及到多重环节的训练, 学生的成绩重过程评价。具体成绩考 核占比为: 文献检索及实验方案的制定, 占比为 $20 \%$; 实验设备、药品的准备, 材料的采购及预处 理, 占比为 $10 \%$; 实验预试、实验过程、实验数据, 占比为 $40 \%$; 论文撰写, 占比为 $30 \%$ 。 


\section{7 结语}

本实验源于科研, 服务于教学, 符合新时期创新人才培养的教改需求。项目的创新点在于: (1) 具有文化育人特色。项目以工夫茶文化为背景, 让学生从茶文化出发提出问题, 将冲泡工艺一个 文化现象转化为一个化学问题, 这不仅让学生得到良好的文化熏陶, 而且利于培养学生在实践中善 于发现问题和解决问题的能力, 以及正确的科学思维习惯。(2) 具有科教融合的特色。项目源于科研 项目, 转化为教学的校本课程, 实验设计富含有趣味性, 实验中文献的检索、方案的制订、实验操 作、数据处理及论文撰写等一系列的实践过程, 有利于培养学生的实践创新能力, 养成良好的创新 思维习惯与科学态度。(3) 本实验充当了基础实验与专业实验的桥梁作用。学生通过本实验的学习, 不仅巩固与拓宽了已学的知识及技能, 而且了解了科学实践的过程及科学研究方法, 为学生后续开 展大学生 “挑战杯” 科技创新项目, 独立完成毕业论文的工作打下了坚实的基础。总之, 教学实践 表明本实验具有良好的育人功能, 且可操作性好, 具有一定的推广价值。

\section{参 考 文 献}

[1] 陈佳雯, 林秀玲, 孙彬妹, 刘少群, 郑鹏. 农业考古, 2021, No. 2, 33 .

[2] 叶汉钟, 蔡汉权, 李粉玲. 福建茶叶, 2013, No. 2, 47.

[3] 陈宗禁. 中国茶叶, 2019, 41 (9), 11.

[4] 罗否, 郭晓园. 食品科学, 2009, 30 (21), 63.

[5] 张祎敬, 宋贤良, 黄苇, 谢怡昕, 周颖君. 食品科技, 2018, 43 (1), 215.

[6] 丁建英, 王晓飞, 张丽, 权英. 南方农业学报, 2018, 49 (2), 340.

[7] 梁萱, 李嘉会, 梁永锋. 中国现代中药, 2018, 20 (6), 698. 